\title{
Isolation and characterization of Xenopus laevis homologs of the mouse inv gene and functional analysis of the conserved calmodulin binding sites
}

\author{
Yukuto Yasuhiko, ${ }^{1, *}$, Koichiro Shiokawa ${ }^{1, *}$, Toshio Mochizuki ${ }^{2, *}$, Makoto Asashima $^{3}$, Takahiko Yokoyama ${ }^{4}$ \\ ${ }^{1}$ Department of Biological Sciences, Graduate School of Science, University of Tokyo, 7-3-1 Hongo, Bunkyo-ku, Tokyo 113-0033, \\ Japan; ${ }^{2}$ Department of Medicine, Kidney Center, Tokyo Women's Medical University, School of Medicine, Japan; ${ }^{3}$ Department of \\ Life Science, University of Tokyo, 3-8-1 Komaba, Meguro-ku, Tokyo 158-8902, Japan; ${ }^{4}$ Department of Anatomy and Developmental \\ Biology, Tokyo Women's Medical College, 8-1 Kawada-cho, Shinjuku-ku, Tokyo 162-8666, Japan
}

The homozygous inv (inversion of embryonic turning) mouse mutant shows situs inversus and polycystic kidney disease, both of which result from the lack of the inv gene. Previously, we suggested that inv may be important for the left-right axis formation, not only in mice but also in Xenopus, and that calmodulin regulates this inv protein function. Here, we isolated and characterized two Xenopus laevis homologs (Xinv-1 and Xinv-2) of the mouse inv gene, and performed functional analysis of the conserved IQ motifs that interact with calmodulin. Xinv-1 expresses early in development in the same manner as mouse inv does. Unexpectedly, a full-length Xenopus inv mRNA did not randomize cardiac orientation when injected into Xenopus embryos, which is different from mouse inv mRNA. Contrary to mouse inv mRNA, Xenopus inv mRNA with mutated IQ randomized cardiac orientation. The present study indicates that calmodulin binding sites (IQ motifs) are crucial in controlling the biological activity of both mouse and Xenopus inv proteins. Although mouse and Xenopus inv genes have a quite similar structure, the interaction with calmodulin and IQ motifs of Xenopus inv and mouse inv proteins may regulate their function in different ways.

Cell Research (2006) 16:337-346. doi:10.1038/sj.cr.7310044; published online 13 April 2006

Keywords: Left-right asymmetry, inv, calmodulin, ankyrin, IQ motif, Xenopus, calcium

\section{Introduction}

Although vertebrates are externally bilaterally sym-

Correspondence: Takahiko Yokoyama

Department of Anatomy, Kyoto Prefecture University of Medicine, 465 Kajiicho, Hirokoji- agaru, Kawaramachi-dori, Kamikyo-ku, 602-0841, Japan Tel: 81-75-251-5303; Fax: 81-75-251-5304;

E-mail: tyoko@koto.kpu-m.ac.jp

*Present addresses: Yukuto Yasuhiko ${ }^{1}$, Koichiro Shiokawa ${ }^{2}$, Toshio Mochizuki $^{3}$

${ }^{1}$ Cellular and Molecular Toxicology Division, National Institute of Health Sciences, 1-18-1 Kamiyoga, Setagaya-ku, Tokyo 158-8501, Japan

${ }^{2}$ Department of Biosciences, School of Science and Engineering, Teikyou University, 1-1 Toyosatodai, Utsunomiya-city, Tochigi prefecture 320-8551, Japan

${ }^{3}$ Department of Medicine II, Hokkaido University Graduate School of Medicine, Kita 15, Nishi 7, Kita-ku, Sapporo 060-8638, Japan

Received 1 Oct 2005 ; revised 14 Nov 2005; accepted 6 Dec 2005, published online 13 Apr 2006 metric, internal organs show a marked left-right (L-R) asymmetry, such as the left-sided presence of stomach and heart and occurrence of more lung lobes on the right side. This L-R asymmetry is genetically determined, and disturbance of the asymmetry often causes serious cardiovascular disease in humans $[1,2]$. Recent progress suggested that primary cilia on the primitive node have an important role in the establishment of the L-R asymmetry. Nodal cilia rotate and make a leftward current [3-5]. This leftward flow subsequently induces asymmetrical gene expression such as nodal and lefty. The mouse mutant called inv (inversion of embryonic turning) shows a constant reversal of the L-R asymmetry in internal organs and develops cysts of the kidney [6]. In inv mutant mouse embryos, the left side expression of nodal and lefty is inverted [7, 8] and abnormal nodal flow was reported [4, 9], indicating that $i n v$ acts upstream of nodal and lefty. The mouse inv gene encodes 1062 amino acids containing 15 tandem repeats of 
the ankyrin motif, two IQ motifs and two nuclear localization signals [10]. In the inv mouse, only the first 91 amino acids of the inv protein remain, but the regions containing the ankyrin repeats as well as the IQ motifs were deleted $[10,11]$. The introduction of inv cDNA completely rescued all the inv phenotypes, not only situs inversus but also cyst formation of the kidney, indicating that the loss of the inv gene caused these two unrelated abnormalities [10]. In humans, a mutation in the inv gene is also responsible for human laterality defects [12] and nephronophthisis (NPHP2) [13]. Thus, the inv gene is essential in establishing normal L-R asymmetry and kidney development in both mice and humans.

Previously, we reported that the mouse inv protein interacts with calmodulin at two sites, and that microinjection of mouse inv mRNA into the right blastomere of two-celled Xenopus embryos resulted in randomized L-R asymmetry in the orientation of visceral organs [14]. Furthermore, the calmodulin binding motif (the IQ motif) is critical for mouse inv mRNA in randomizing cardiac orientation, and $\mathrm{Ca}^{2+}$ regulates the interaction between calmodulin and the mouse inv protein.

In this study, we isolated two distinct full-length cDNAs for Xenopus laevis inv homologs (Xinv-1 and Xinv-2, accession numbers AF321228 and AF321229 respectively), examined their expression and performed functional analysis of the conserved IQ motifs.

\section{Materials and methods}

\section{Cloning of Xenopus inv homologs}

Degenerative oligonucleotides were designed from regions encoding conserved ankyrin repeats of mouse and human inv proteins. Total RNAs of adult Xenopus kidney and liver were used as a template for cDNA synthesis. These RNAs were reverse-transcribed with Superscript II (Invitrogen, Carlsbad, California), and used as PCR templates. An amplified product was subcloned into the pGEM Tvector (Promega, Madison, WI) and sequenced. The product encoded multiple ankyrin repeats that were $70 \%$ identical in amino-acid sequence to mouse inv ankyrin repeats 3-10. The fragment was labelled with ${ }^{32} \mathrm{P}$-dCTP using an oligopriming kit (Amersham Pharmacia Biotech, Uppsala Sweden) and used as a probe to screen a Xenopus oocyte cDNA library (Clontech ZL5000a, Clontech, Mountain View, CA, USA). The first screening gave us four inv-related cDNA fragments. Sequences showed that three of these fragments encoded the same gene and were named Xinv-1, and the fourth cDNA was named Xinv-2. We screened at least half a million clones at each library screening.

To obtain a full coding sequence of the Xinv genes, we screened cDNA libraries, including two oocyte cDNA libraries (Clontech ZL5000a and ZL5001T), the kidney and liver cDNA libraries (Stratagene, La Jolla, CA, USA) and the stage 11.5 embryo cDNA library (a gift from Dr Bruce Blumberg). Rapid Amplification of cDNA Ends (RACE) was also performed to obtain the 3' end of Xinv-1 using oocyte cDNA as a template.

\section{Northern hybridization and RT-PCR}

For Northern analysis, polyA RNAs were purified from total RNAs obtained from oocytes and liver. Five mg of polyA RNAs were electrophoresed and blotted onto nylon membranes (Amersham Pharmacia Biotech). Membranes were hybridized with a ${ }^{32} \mathrm{P}$-labelled Xenopus inv probe $(X i n v-1)$ and washed with $2 \times \mathrm{SSC}, 0.1 \%$ SDS at $60{ }^{\circ} \mathrm{C}$ and exposed to X-ray film for 1-7 days.

For RT-PCR, RNA was extracted from embryos at various stages by the AGPC method. Reverse transcription was performed using MMLV reverse transcriptase (Invitrogen) following the manufacturer's protocol. Primers specific to each Xinv clone were as follows:

Xinv-1 forward: 5'-GCT GGT GGAAAA GGA GAAAGC TAG AA-3'

Xinv-1 reverse: 5'-CCT ACA TTA CAA CAC TGG AGA GCG CA-3'

Xinv-2 forward: 5'-CCT ACA TTA CAA CAC TGG AGA GCG CA-3'

Xinv-2 reverse: 5'-CTA CAC CAC ACC AAA CGT CAA TTG AAA-3'.

PCR reaction was performed using Ex Taq (Takara, Kyoto).

We amplified $\beta$-actin to verify the RT reaction. Primers for $\beta$-actin amplification were actinF: 5'-GGA CTT GGC TGG ACG TGA CCT GAC-3', actinR: 5'-CAG ACT CAT CAT ACT CCT GCT TGC TG-3' 18 cycles of PCR were performed.

\section{Whole-mount in situ hybridization}

The plasmid pYY94 contained 1453 bp of 3 ' terminal region of Xinv-1 ORF (open reading frame) in pBluescript SK(-) (Stratagene, La Jolla, CA, USA). The plasmid was linearized at either the Not I site (for the antisense probe) or the Xho I site (for the sense probe) and subjected to in vitro transcription using T7 or T3 polymerase to produce an antisense or sense probe, respectively, in the presence of digoxigenin-modified UTP. Albino $X$. laevis embryos at various stages were fixed in MEMPFA (0.1 M MOPS pH 7.4, 2 mM EGTA, $1 \mathrm{mM} \mathrm{MgSO}_{4}, 4 \%$ paraformaldehyde) and processed for whole mount in situ hybridization as described previously [15]. Coloring reaction was performed overnight at $4{ }^{\circ} \mathrm{C}$, then overnight at room temperature using BM purple (Roche Diagnostics, Basel, Switzerland).

DNA construction, site-directed mutagenesis, in vitro transcription for $m R N A$ injection and in vitro translation

The ORFs of Xinv-1 and - 2 were PCR-amplified and ligated into the transcriptional vector pCS2+ [16] between the EcoR I and Stu I sites using a ligation kit (Takara). The sequence was confirmed not to have a mutation. For Xinv- 1 transcription, we substituted the T3 promoter for the SP6 promoter of pCS2+. The plasmids, pYY158 (Xinv-1) and pYY159 (Xinv-2), were linearized at the Not I site, and transcribed with T3 RNA polymerase using MEGAscript kit (Ambion).

Mouse inv cDNA (pGEM-mINV) [10] was digested with EcoR I (Takara) and the $3 \mathrm{~kb}$ fragment obtained was ligated into the $E c o R$ I site of pCS2+. The resultant plasmid pYY34 was linearized at the Not I site and transcribed with SP6 RNA polymerase. Site-directed mutagenesis of Xenopus inv IQ motifs was performed using GeneEditor kit (Promega). The mutated ORFs were subcloned to pCS2+ (modified its promoter from SP6 to T3) and transcribed with T3 RNA polymerase. To obtain GFP mRNA, pbGFP/RN3P [17] was linearized with SfiI and transcribed with T3 RNA polymerase. All 
in vitro transcription was performed in the presence of a cap analog (New England Biolabs).

Two cDNA clones, Xinv-1 and -2, were subjected to in vitro translation using rabbit reticulocyte lysate (Promega) in the presence of ${ }^{35}$ S-methionine. Subsequently, lysates were analyzed with SDSPAGE. The gel was dried and examined by autoradiography.

\section{Yeast two-hybrid assay}

The following plasmids were used to examine the interaction with calmodulin in the MATCHMAKER yeast two-hybrid system (Clontech):

GBT-158 (Xinv-1 full-length cDNA)

GBT-182 (IQ1 mutated in Xinv-1)

GBT-183 (IQ2 mutated in Xinv-1)

GBT-184 (IQ1 and IQ2 mutated in Xinv-1)

The vector was derived from the $\mathrm{pGBT}$ vector (Clontech). Plasmids were introduced into the Y187 yeast strain with pCaM8 that contained the calmodulin gene fused to an activating domain for the yeast two-hybrid assay. Colonies, grown on minimal media lacking tryptophan and leucine, were tested for $\beta$-galactosidase activity with a colony-lift filter assay.

\section{Microinjection of synthetic $m R N A$}

Unfertilized eggs of $X$. laevis were manually ovulated from gravid females that had been injected with a human chorionic gonadotropic hormone, gonatropin (Teikoku Zoki, Kawasaki Kanagawa). Eggs were artificially fertilized, dejellied in $2 \%$ cysteine- $\mathrm{HCl}(\mathrm{pH} 8.0)$ and kept in $0.1 \times$ Steinberg's solution [18]. The mRNAs to be tested were microinjected at a dosage of $2000 \mathrm{pg} / \mathrm{embryo}$ unless otherwise noted, together with GFP mRNA (200 pg/embryo in all the experiments) in $10 \mathrm{nl}$ of distilled water. As injection of $5 \mathrm{ng} \mathrm{mRNA} /$ embryo resulted in a frequent occurrence of malformed embryos (abnormal cleavage and gastrulation failure), we decided to inject $2 \mathrm{ng}$ of $i n v$ mRNAs per embryo. Microinjection was performed at the late two-cell stage or the early four-cell stage. The mRNA solutions were injected into the right or left half of each embryo in the animal hemisphere at the presumptive dorsal and ventral regions. All of the embryos were placed in $1 \times$ modified Barth's solution (MBS), containing 3\% Ficoll 400 and $50 \mu \mathrm{g} / \mathrm{ml}$ gentamycin during microinjection. Injected embryos were kept in $1 \times \mathrm{MBS}$ until stage 7 [19], and subsequently transferred into $0.1 \times$ Steinberg's solution for further culture. Embryos were kept at $23{ }^{\circ} \mathrm{C}$ in $0.1 \times$ Steinberg's solution, containing $50 \mu \mathrm{g} / \mathrm{ml}$ gentamycin, before further observations were made. Embryos were cultured until stage 22 [19], and grouped into left-side injected and right-side injected embryos according to the distribution of the GFP signal under a fluorescent microscope (Olympus Model BHS-RFK, with a filter set for FITC observation). At stage 45 , embryos were paralyzed with Tricaine $(0.1 \%$ in $0.1 \times$ Steinberg's solution) and the orientations of heart and gut were examined under a dissection microscope. Statistical analyses were performed using a standard $\chi^{2}$ test, and $p<0.05$ was considered a significant difference.

\section{Results}

Two Xenopus inv homologs contain conserved ankyrin repeats and two IQ motifs

Two Xenopus cDNA clones, named Xinv-1 and -2, contained an open reading frame (ORF) homologous to mouse inv, and encode 1007 and 1002 amino acids, respectively (Figure 1A). Both ORFs started with an ATG initiation codon that agrees with the Kozak rule [20], and have an in-frame stop codon in the 3 ' end. The clones are $89 \%$ identical to each other in amino-acid sequence. Nucleotide sequences of ORFs of Xinv-1 and Xinv-2 are 58.2\% and $57.8 \%$ identical to that of the mouse inv ORF. At aminoacid level, Xinv-1 and Xinv-2 have 50\% and 52\% overall amino-acid identity to mouse inv. Both Xinv-1 and Xinv-2 have 15 repeats of the ankyrin motif in the N-terminus (a yellow box in Figure 1A), and two calmodulin-binding IQ motifs, named IQ1 and IQ2 (16 amino-acid residues), that are highly homologous to those of mouse and human inv genes. Amino-acid sequences of the ankyrin repeats of Xinv- 1 are $73.4 \%, 71.8 \%$ and $74.5 \%$ identical to that of mouse, chick and zebrafish inv, respectively. Amino-acid sequences of the ankyrin repeats of Xinv-2 are $74.7 \%$, $75.5 \%$ and $72.7 \%$ identical to that of mouse, chick and zebrafish inv, respectively. A search in the $X$. tropicalis database identified a sequence that was homologous to Xinv-1 and -2. The sequence encodes 15 repeats of the ankyrin motif and two IQ motifs. The sequence has $83.5 \%$ and $83.1 \%$ overall amino-acid identity to Xinv-1 and -2 , respectively. We could not find other related sequences in mouse, human and X. tropicalis databases. IQ1 is just behind the ankyrin repeats, and IQ2 is located near the C-terminus (red boxes in Figure 1A). We also found two basic amino-acid residues, one next to IQ1 and the other at the N-terminal side of IQ2, conserved between mouse and Xenopus (blue boxes), which are named Basic Region (BR)-1 and -2 , respectively. There is a conserved hydrophobic region (designated $\mathrm{HR}$ ) in the amino-terminal side of BR2 (green box in Figure 1A). Despite the general similarity of the deduced protein structure, Xinv-1 has only one monopartite (SV40T antigen type) nuclear localization signal (NLS), and Xinv-2 has no typical NLS, while there are two typical bipartite (nucleoplasmin type) nuclear localization signals (NLS) in mouse inv protein (Figure1B). These structures are well conserved among species (Figure 1B). Figure 2 shows a phylogenetic relationship among mouse, human, chick, zebrafish and Xenopus (tropicalis and two laevis) inv genes.

\section{Xinv-1 is expressed maternally and in early embryos of Xenopus}

The Northern hybridization analysis detected two signals of Xinv mRNA (about 4.4 and $5.8 \mathrm{~kb}$ ) in the ovary. Only a larger band (about $5.8 \mathrm{~kb}$ ) was detected in the liver (Figure3A). As we obtained two Xinv cDNAs, we made specific primers in their 3' ends to distinguish $X i n v-1$ from Xinv-2 mRNAs, and examined their expression. The signal for Xinv-1 was constantly obtained in RNA from the ovary, 
A Xinv1(x.laevis).sea Xinv2(X.loevis).sea invs (X.tropicalis).sea inv(M.musculus).sea invs(H.sapiens). sea

Xinv1(X.laevis).sea Xinv2( $X$. laevis). sea invs (X.tropicalis).sea inv(M.musculus). sea invs(H.sapiens). sea

Xinv1(X.laevis).sea Xinv2(X.loevis).sea invs (X.tropicalis).sea inv(M.musculus). sea invs(H.sapiens). sea

Xinv1(X.laevis).sea Xinvz(X.laevis).sea invs (X.tropicalis).sea inv(M.musculus). sea invs (H.sapiens). sea

Xinv1(X.laevis).sea Xinvz(X.laevis).sea invs (X.tropicalis).sea inv(M.musculus). sea invs(H.sopiens). sea

Xinv1(X.laevis).sea Xinv2(X.laevis). sea invs (X.tropicalis).sea inv(M.musculus).sea invs(H.sopiens). sea

Xinv1(X.laevis).sea Xinvz(X.laevis).sea invs (X.tropicalis).sea inv(M.musculus).sea invs(H.sapiens). sea

Xinv1(X.laevis).sea Xinvz(X.laevis).sea invs (X.tropicalis).sea inv(M.musculus) sea invs (H.sapiens). sea

Xinv1(X.laevis).sea Xinvz(X.laevis). sea invs (X.tropicalis). sea inv(M.musculus). sea invs(H.sapiens).sea

Xinv1(X.laevis).sea Xinv2(X.laevis).sea invs(X.tropicalis).sea inv(M.musculus).sea invs(H.sopiens). sea

Xinv1(X.laevis).sea Xinv2(X.laevis).sea invs (X.tropicalis).sea inv(M.musculus). sea invs(H.sopiens). sea

Xinv1(X.laevis).sea Xinv2(X.laevis).sea invs (X.tropicalis). sea inv(M.musculus). sea invs(H.sopiens). sea

Xinv1(X.laevis).sea Xinvz(X.laevis).sea invs (X.tropicalis).sea inv(M.musculus). sea invs(H.sopiens). sea

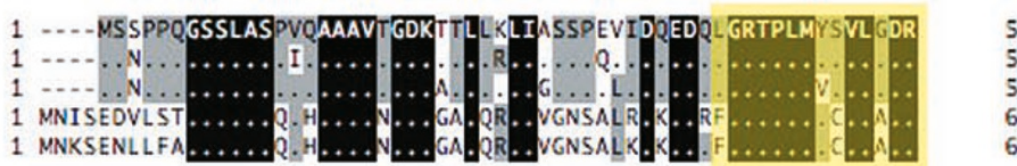

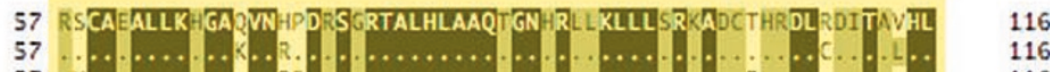

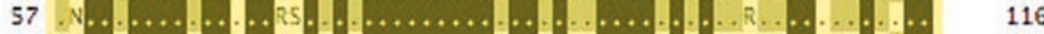

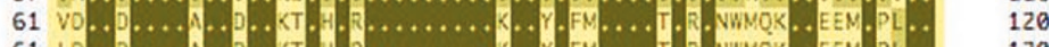

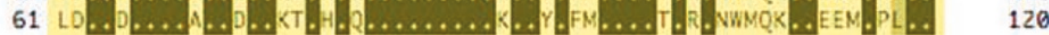

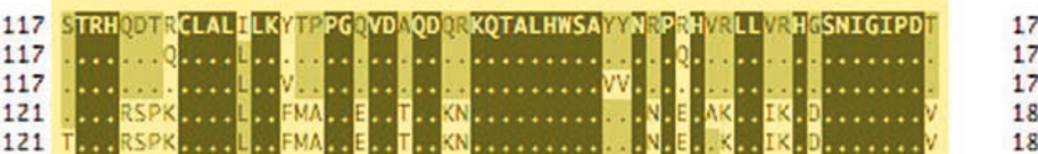

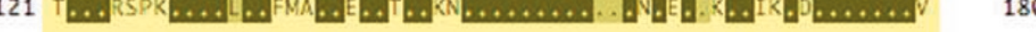

177 EGKIPLHIKAAGHKDP EAMLTVRCLLERAPTESLLNWQDVEGRTPLHLAVGDGKREVVRLL 236

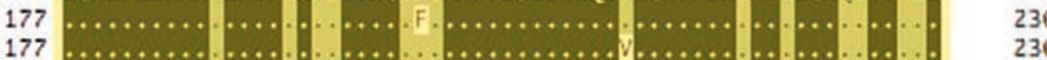
$181 \ldots \ldots \ldots \ldots$. . . S.VH .... I. D . . . . . . . . . . F . . . . LT . DV.

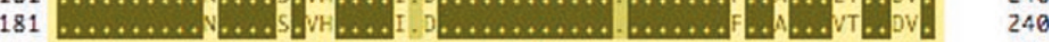

237 ISYRGCNVAPY DNLFRTPLHWAALLGYTPIA HLLLET JVSPNIPSDSQGATPLHYAAQON 296

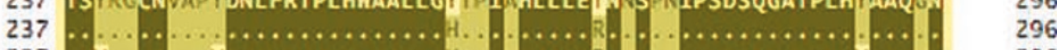

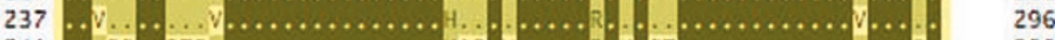

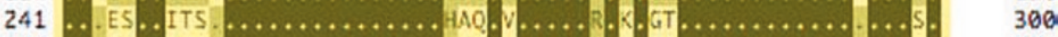

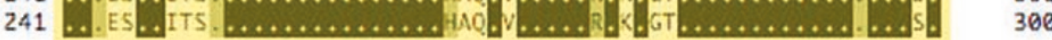

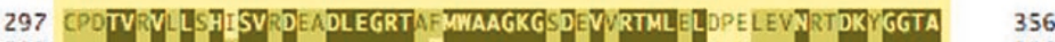
297 ............................................... 356

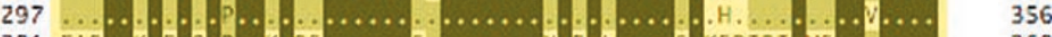

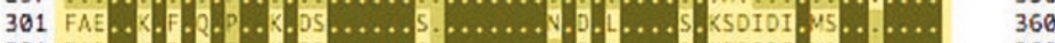
301 FAE..K.F.K.P..K.DS.......S.............. S.KSDIDI. MA..... 360

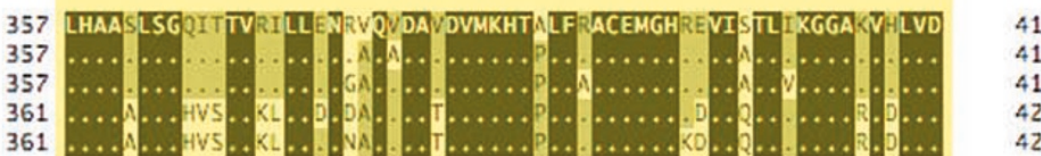

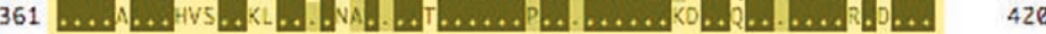
417 KDG इSPLHWAALGGNA YVCQILIEN IINPDAQDYEGRTPLQCAAYGGYI GCMEVLMENKA 47

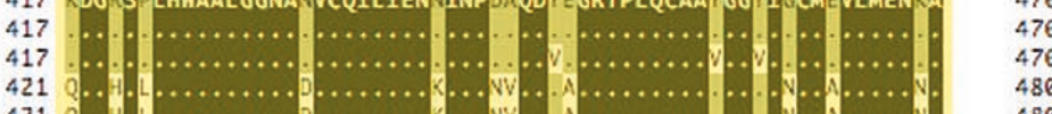

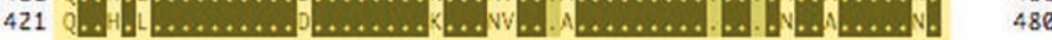
477 DPNIQDKNGRTALHWSCNNGYLDAVKLLLGYS RFPNQMENT EERYTPLDYALLGGHQEVI 536

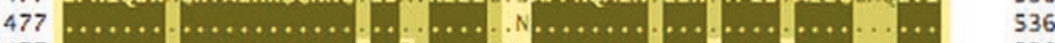

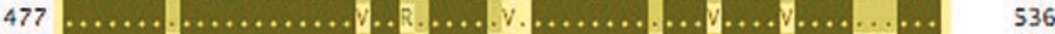

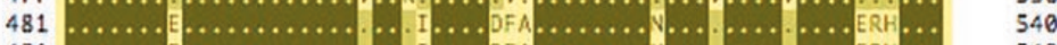

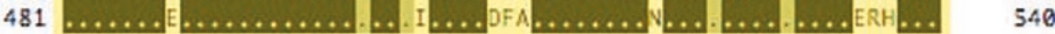

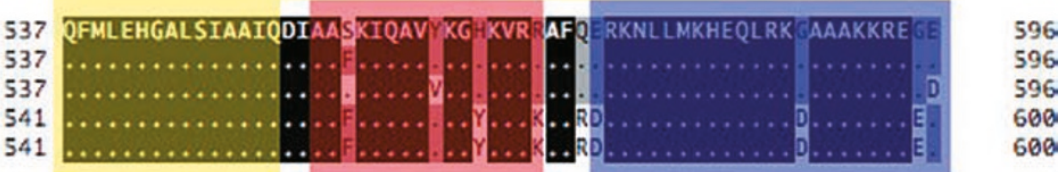
597 VROXKGVGOTEGKOKDENHVMRODKSNEHI-ONEVMREWYGEETGRAEDRKEEHQEEÑNN 597 ...V....K.... AOSME..N....Q.IK...VH. .Q. . AS.N. ...GK.R.... 654

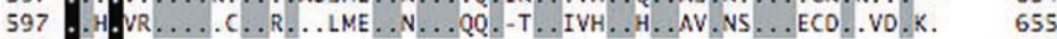

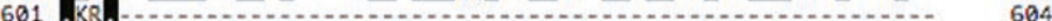
601 KR. 604

656 IEPKOLKHSKHMEONSKSIAKNOKRAGHIQSSPIEHVHTNSIQTRMSPSRTSISHSSPLG 715

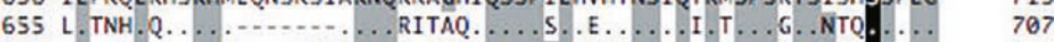

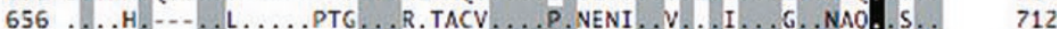
605 . 605 ................. EAEQQK. R-.RSPDS-CRPQA-LPCLPS.QDVP. RQSR 638

716 NETPKNMYWDONPTQNNTQPRRTSRPQI ESPNII VHRIEDLVQKESRRKSHREERKGSHR 775

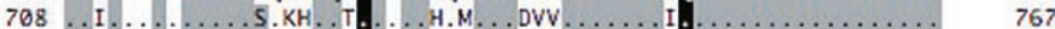

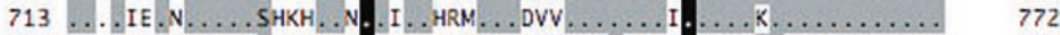
645 TPSKQPPASHTVQSPOPEHS. -LPGRCPGRASQGDSS. - -DL.GTAS..PSETPIEHCRG 701 639 APSKQPPAGNVAQGPEPRDS.GSPGGSLGGALQKEQHVSSDL.GTNS. RPNETA.EH.KG 698 
Xinv1( $X$. laevis). sea Xinvz( $X$. laevis). sea invs (X.tropicalis). sea inv( $M$.musculus). sea invs (H.sapiens). sea

Xinv1(X. laevis). sea Xinvz(X.laevis). sea invs (X.tropicalis). sea inv(M.musculus). sea invs (H.sapiens). sea

Xinv1 (X. laevis). sea Xinvz( $X$. laevis). sea invs (X.tropicalis). sea inv(M.musculus). sea invs(H.sapiens). sea

Xinv1(X. laevis).sea Xinvz(X. laevis). sea invs (X.tropicalis). sea inv(M.musculus). seo invs (H.sapiens). sea

Xinv1(X.laevis).sea Xinvz(X. laevis). sea invs (X.tropicalis). sea inv(M.musculus).sea invs (H.sapiens).sea

Xinv1(X. laevis).seq Xinvz(X. laevis). sea invs (X.tropicalis). sea inv(M.musculus). sea invs (H.sapiens). sea

Xinv1(X.laevis). sea Xinvz(X. laevis). sea invs (X.tropicalis). sea inv(M.musculus). sea invs(H.sapiens). sea

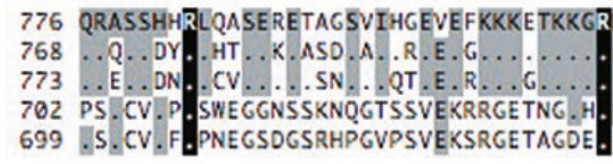

TAAGTSKIRAS- - GEAGRLSQSE V.V.P. E. CCK.GC.K.

I. T. ...TG. C- -S.GV.....

CEE.P.SA.QPLCTGS. .PAEKG

-C.K. KGFVKQPSCIRVAGPOEKG

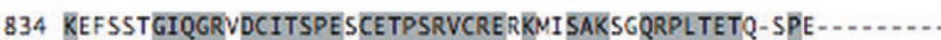

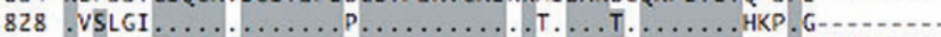

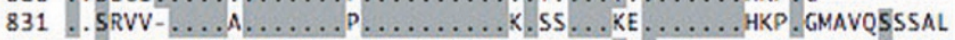

762 EDS. PAVASASOQDHPRKPNKRODRAARPRGASOKRRTH. LRD-RCSPAGSSRPGSAKGE

758 EDSRRA. ASLPPHDSHWKP. RRHDTEPKAKCAPQKRRTQELRGGRCSPAGSSRPGSARGE

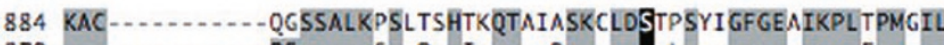

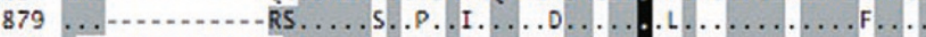

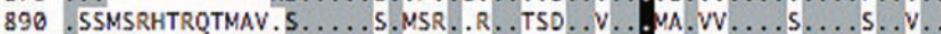

821 V...ADQSSLHRHTPRS KVTQDKLIGGVSSGLPLSTEASR. GCKQL . . . . YEDICASPET

818 AVHAGQNPPHHRTPRNKVTQAKLTGGLYSHLPQSTEE R. GARRLETSTLSEDFQVSKET

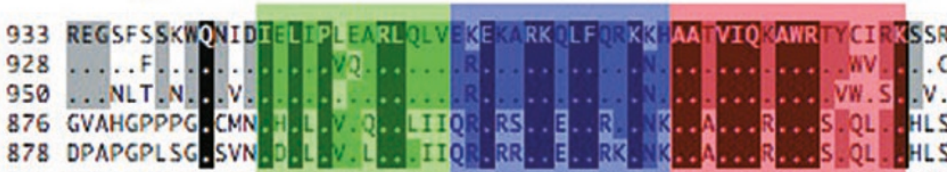

993 KTRHSHLRNNPRAMV

$1010 \mathrm{~T}$

L. LKQLGAREVLRCTQVCTALLLQVWRKELELKFPKSISVSRTSKSPSKGSSATKYAR
MKOLGAGDVDRWRQESTALLLOVWRKELELKFPOTTAVSKAPKSPSKGTSGTKSTK

833

827

830

761

757

883

878

889

820

817

932

927

949

875

877

992

987

1009

935

937

1007

1002

1024

995

997

1007

1002

(2.

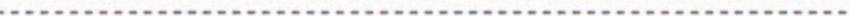

996 HSVLRQIYGCSQEGKGHHPIKSSKAPAVLHLSSVNSLQSIHL-ONSGRSKKFSYNLQPSS

998 HSVLKOIYGCSHEGKIHHPTRSVKASSVLRLNSVSNLQCIHLLENSGRSKNFSYNLQSAT

1007

1002

1024

1054

1057

1007

$1002 \ldots$

1024

1055 QSKNKPKL

1058 QPKNKTKP
1007

1002

1024

1062

1065

B

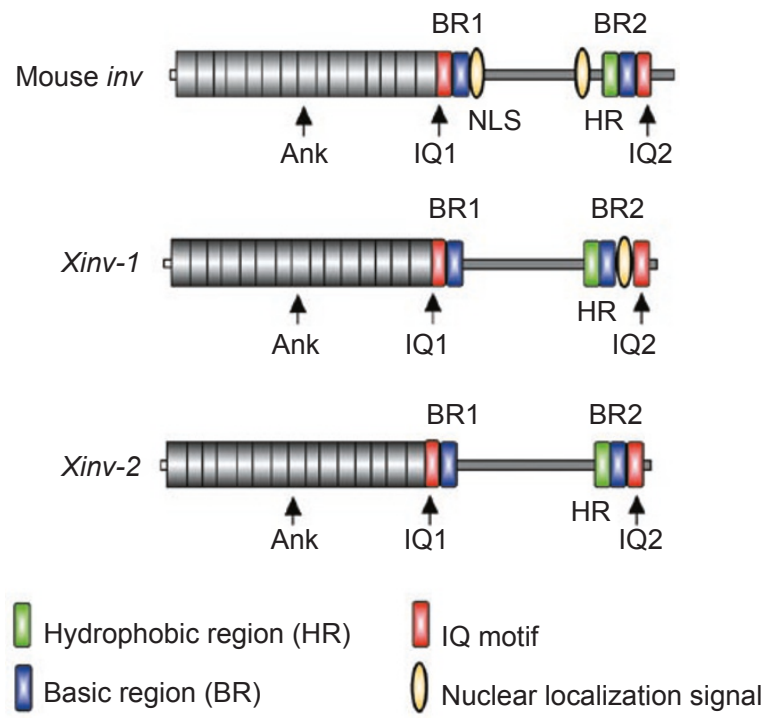

Figure 1 (A) Alignment of putative Xenopus laevis inv amino-acid sequences along with mouse, chick (Gallus gallus) and zebrafish (Danio rerio), and putative $X$. tropicalis inv protein sequences. Xinv-1, Xinv-2, X. tropicalis inv, zebrafish inv, chick inv and mouse inv proteins are 1007, 1002, 1020, 1021, and 1269 and 1138 amino acids, respectively. The conserved motifs are indicated as follows. Yellow boxes: ankyrin repeats, red boxes: IQ motifs, blue boxes: conserved basic regions (BRs), green box: conserved hydrophobic region (HR). IQ motifs and BRs were numbered from the N-terminal (IQ1, 2 and BR1, 2, respectively). (B) Schematic description of the structure of mouse and Xenopus inv proteins. 


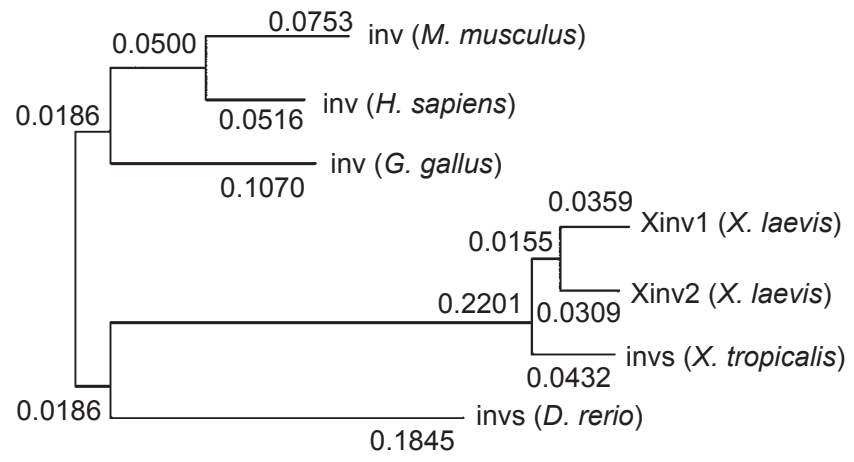

Figure 2 A phylogenic tree of inv related genes. The tree is constructed by the neighbor-joining method [21] using the amino-acids sequences of ankyrin repeat domains. Values indicate expectation of nucleotide substitution on each residue.

cleavage stage embryos through stages 3-27 (Figure 3B). Although the signal for $\beta$-actin was detected at 18 PCR cycles, the signal for Xinv-2 was not detected from the unfertilized egg (ovary) to the hatching stage (St.33), even at 50 PCR cycles (Figure 3B). The detection of Xinv signals in the ovary suggested a possibility that the inv message is maternally provided in the egg.

Whole-mount in situ hybridization (Figure 3C) showed that the Xinv mRNA signal was present in the morula (St. 7) to the tailbud stage. After the neurula stages, Xinv signals were detected in the dorsal side of embryos. In the tailbud stage (St. 22), Xinv signals were also in the dorsal side with a little anterior bias. No obvious asymmetrical pattern was observed in the examined embryos. Throughout the stages, the signal was not detected using a sense probe.

\section{A single amino-acid substitution of isoleucine to glutamic} acid eliminates calmodulin-inv protein interaction

We reported that the region encoding the IQ motifs plays a crucial role in the activity of mouse inv mRNA to randomize the L-R asymmetry of Xenopus embryos by right-sided mRNA microinjection [14]. Sequence analysis shows that Xenopus inv proteins have IQ motifs that are homologous to mouse inv protein (Figure1 A and 4A), and yeast two-hybrid assay showed that Xinv-1 protein also
A

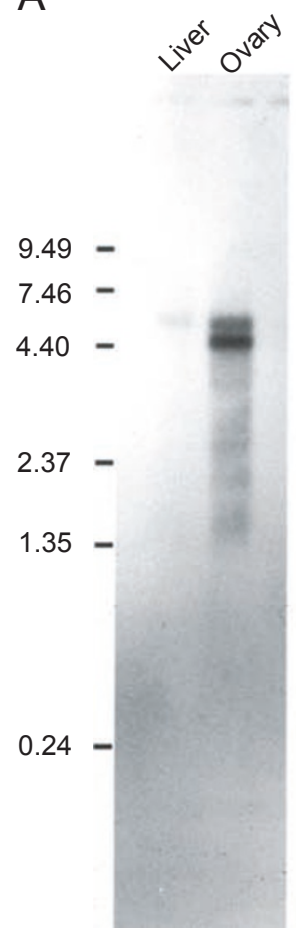

C

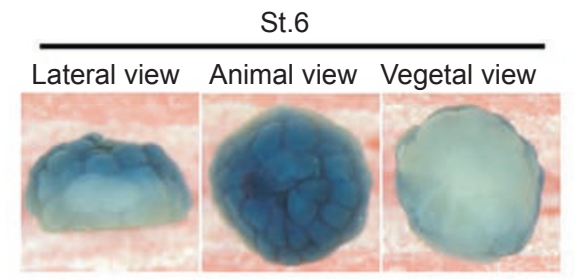

St.14

B

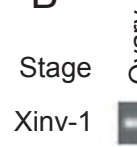

Xinv-2

$\beta$-actin

Xinv-1 RT(-)

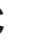

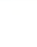

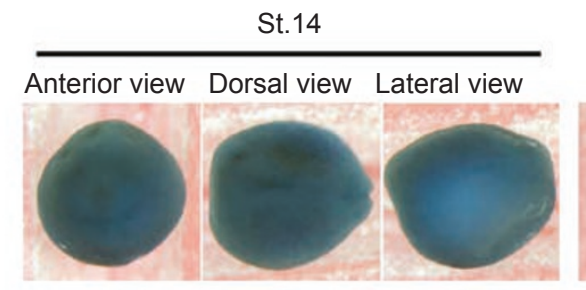

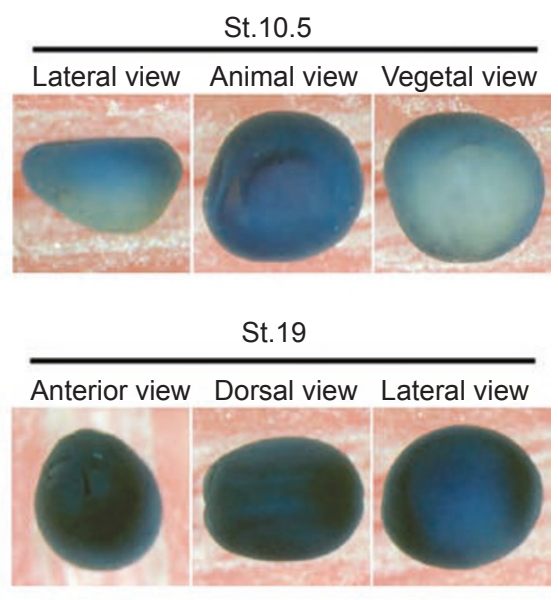

Figure 3 (A) Northern hybridization for Xenopus inv cDNA expression. In all, $2 \mu \mathrm{g}$ of polyA+RNA is loaded in each lane, and exposed for 7 days at $-80^{\circ} \mathrm{C}$. A $4.4 \mathrm{~kb}$ transcript (arrowhead) was detected in ovary while not in liver. Size markers (in kb) are shown in the left side of the autoradiograph. (B) Temporal expression patterns of Xenopus inv examined with RT-PCR. Xinv-2 is not detected by PCR with up to 50 cycles, while Xinv- 1 is detected at 46 cycles. RT-PCR with $\beta$-actin specific primers was carried out in parallel to control the amount of input RNA. RT (-), RT-PCR without reverse transcriptase. Stages are shown over each lane. (C) Expression pattern of Xinv from stage 6 to stage 27 shown by whole-mount in situ hybridization using digoxigenin-labeled Xinv-1 cRNA as a probe. No significant unilateral expression of Xinv is detected. 


$\begin{array}{lll}\text { A } & \text { Xinv-1 IQ1 } & \text { AASKIQAVYKGHKVRR } \\ \text { IQ1E } & \text { AASKEQAVYKGHKVRR } \\ & \text { Xinv-1 IQ2 } & \text { AATVIQKANRTYCIRK } \\ \text { IQ2E } & \text { AATVEQKAWRTYCIRK }\end{array}$

B Bait (Xenopus inv)

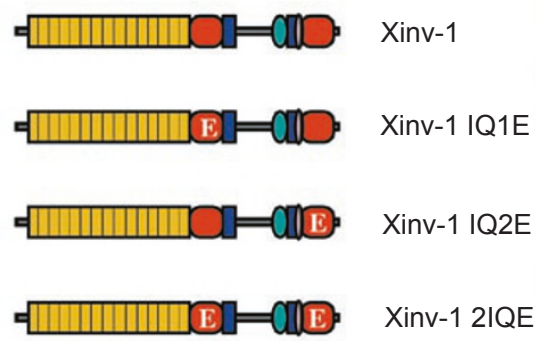

Figure 4 (A) A single amino-acid substitution of isoleucine (I) by glutamic acid (E) eliminates calmodulin-inv protein interaction. (B) Description of the mutated Xinv IQ motifs. Substitution of isoleucine (I) with glutamic acid (E) is indicated in red. IQ motif mutated Xenopus inv's lose calmodulin-binding activity. The interaction between a wild-type Xinv protein or mutated Xinv proteins and calmodulin or SV40 large T antigen (CaM and SV40T, shown in the upper row as 'prey') was assayed using a yeast two-hybrid system. A schematic description of the constructs indicated in the left column as 'bait'. The white letters ' $E$ ' in IQ motifs (depicted as red blocks) represent the amino-acid substitution. In this experiment, wild-type Xinv proteins or mutated Xinv proteins were used as baits. Blue staining indicates the interaction between bait and prey. Only wild-type Xinv-1 shows calmodulin binding activity. SV40 large $\mathrm{T}$ antigen was a prey as a negative control of the yeast two-hybrid assay, and no interaction was observed.

interacts with calmodulin (Figure 4B).

To verify that Xenopus inv protein interacts with calmodulin at the IQ motifs, we performed site-directed mutagenesis at the regions encoding IQ motifs (Figure 4A). We made three mutant constructs of Xinv-1, containing a single amino-acid substitution from isoleucine (I) to glutamic acid (E) in one of their IQ motifs or both (Figure 4A). Xinv-1/IQ1E, Xinv-1/IQ2E and Xinv-1/2IQE contain the mutation in IQ1, IQ2 and in both IQ1 and IQ2 regions, respectively (Figure 4B, left column). A full-length ORF of these mutant constructs were cloned into yeast expression vector, and a two-hybrid assay was performed using mouse calmodulin $(\mathrm{CaM})$ as prey. The yeast two-hybrid assay indicated that all these mutants had lost their calmodulinbinding activity (Figure 4B). Interestingly, mutation in either one of the IQ motifs sufficiently eliminated the whole calmodulin binding activity of the Xinv protein, indicating that the Xinv protein-calmodulin interaction needs two intact IQ motifs.
IQ-mutated but not wild-type Xinv mRNA injection reverses cardiac orientation

Microinjection of mouse inv mRNA into the right blastomere of two-cell stage Xenopus embryos causes randomization of L-R asymmetry and Xnr- 1 expression pattern [14]. To examine whether Xinv mRNA has the same effect on L-R asymmetry, we injected synthetic mRNA of Xinv-1 (total 2 ng per embryo) into one of the blastomeres of twocell-stage Xenopus embryos with GFP mRNA (Figure 5). Unexpectedly, the microinjection of Xinv-1 mRNAs did not cause an $\mathrm{L}-\mathrm{R}$ inversion (Figure 6). We performed in vitro translation experiment of Xinv-1 and Xinv-2 to verify that Xinv mRNAs were translated, using rabbit reticulocyte lysate in the presence of ${ }^{35} \mathrm{~S}$-methionine. Resulted lysates were analyzed with SDS-PAGE and autoradiograph, giving signals at the expected molecular size (not shown).

However, when the IQ mutated Xinv mRNAs were microinjected into the right blastomere of two-cell stage Xenopus embryos, the reversal of cardiac orientation was seen in a significant number of the embryos (Figure 6), though the percentage of cardiac reversals was lower than that caused by mouse inv, 18\% (Xinv-1/2IQE) to $22 \%$ (Xinv-1/IQ2E). Throughout the experiment, left-sided microinjection had no effect on the cardiac reversal rate when compared with noninjected siblings (not shown).

\section{Discussion}

In the present study, we identified two $X$. laevis genes that were homologous to the mouse inv gene. The overall similarity between mouse and Xenopus inv's seems to be relatively low, but there are some distinct conserved domains between them: 15 ankyrin repeats, two CaM-binding motifs and NLSs (though NLS is absent in Xinv-2). These features are highly conserved among previously identified inv homologs of various kinds of vertebrates (mouse, chick and zebrafish). In addition, a search in the $X$. tropicalis database identified a sequence that was more than $80 \%$ identical to the two $X$. laevis genes. Although pylogenetic analysis showed that the distance between mouse and Xenopus inv genes is larger than that between mouse and zebrafish inv genes, this is not significant. Therefore, it is unlikely that the cloned genes are psedoalleles of the inv gene. These results suggest that the genes are $X$. laevis inv genes, and a possibility that their biological functions are shared.

The temporal expression pattern of Xinv- 1 indicated that Xinv mRNAs exist maternally and are expressed constantly during embryogenesis, similar to that of mouse inv [22]. Whole-mount in situ hybridization showed that Xenopus inv mRNA expresses evenly in both left and right sides of embryos. These results are also consistent with the previous observation in mouse embryos [10]. Although the 


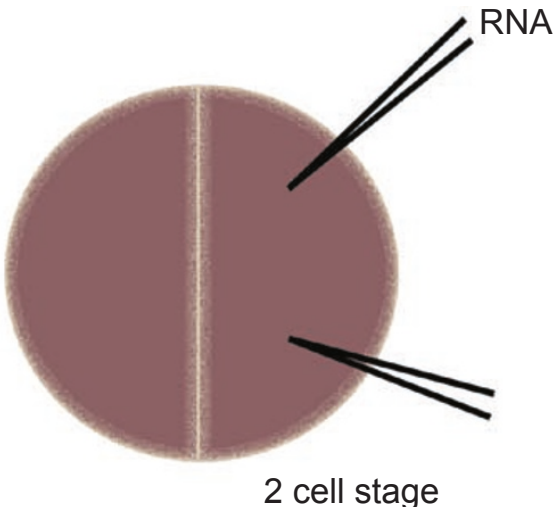

A
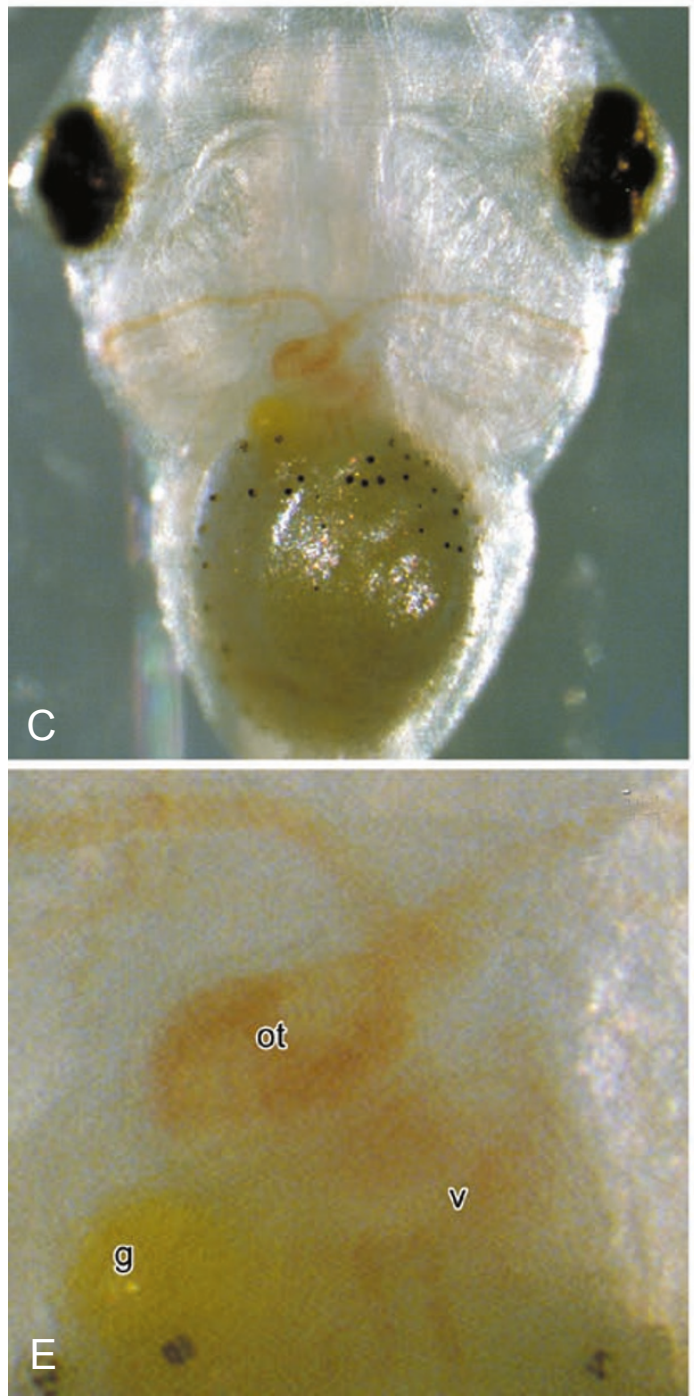
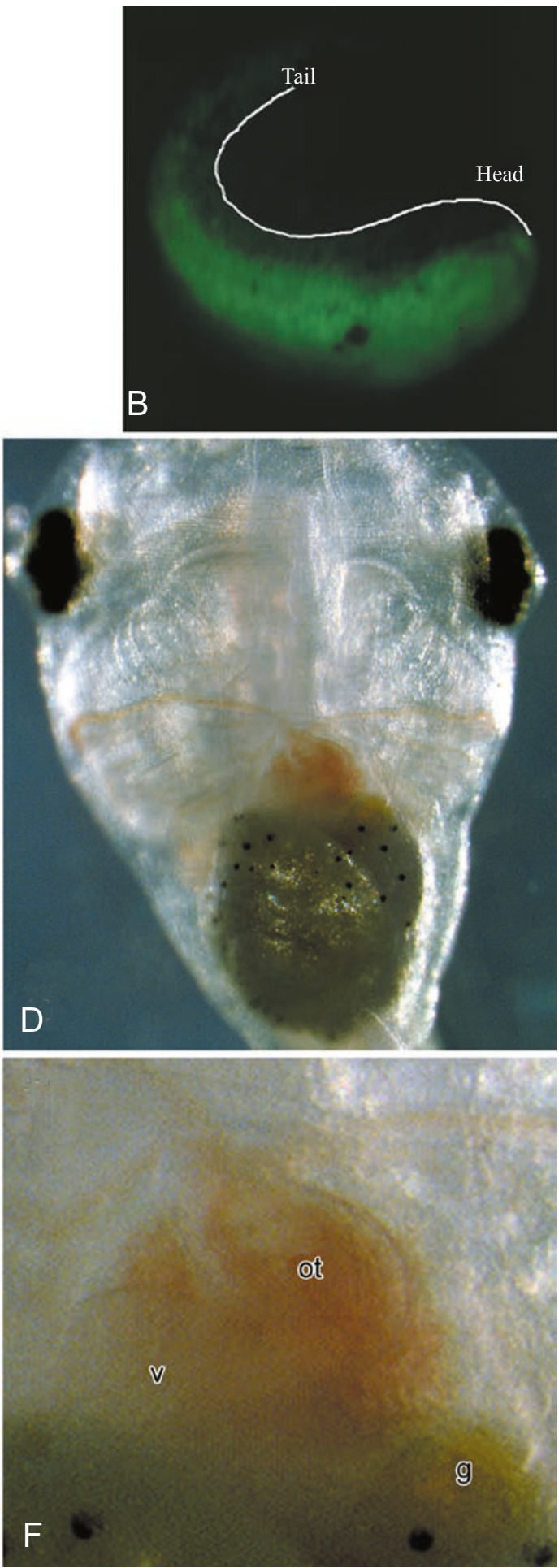

Figure 5 Injection of synthetic mRNAs into the blastomere of the two-cell-stage Xenopus embryo and randomization of the L-R asymmetry. (A) Microinjection was performed at two-cell or early four-cell stage, and mRNA solutions were injected into the left or right half of the embryos. (B) Dorsal view of injected embryo observed under a fluorescent microscopy. GFP fluorescence occurs in the right half of the embryo. The injected embryos were divided into right- and left-injected groups according to the occurrence of GFP fluorescence at St. 23-24. (C) A noninjected embryo showing normal visceral L-R asymmetry. (D) An example of reversal of L-R asymmetry. (E) and (F) The outer appearance of normal heart and gall. Abbreviations: ot, outflow tract; v, ventricle; g, gall bladder. Inverted L-R asymmetry of heart and gall in the embryo. Note that the positions of outflow tract and gall bladder are completely inverted. 

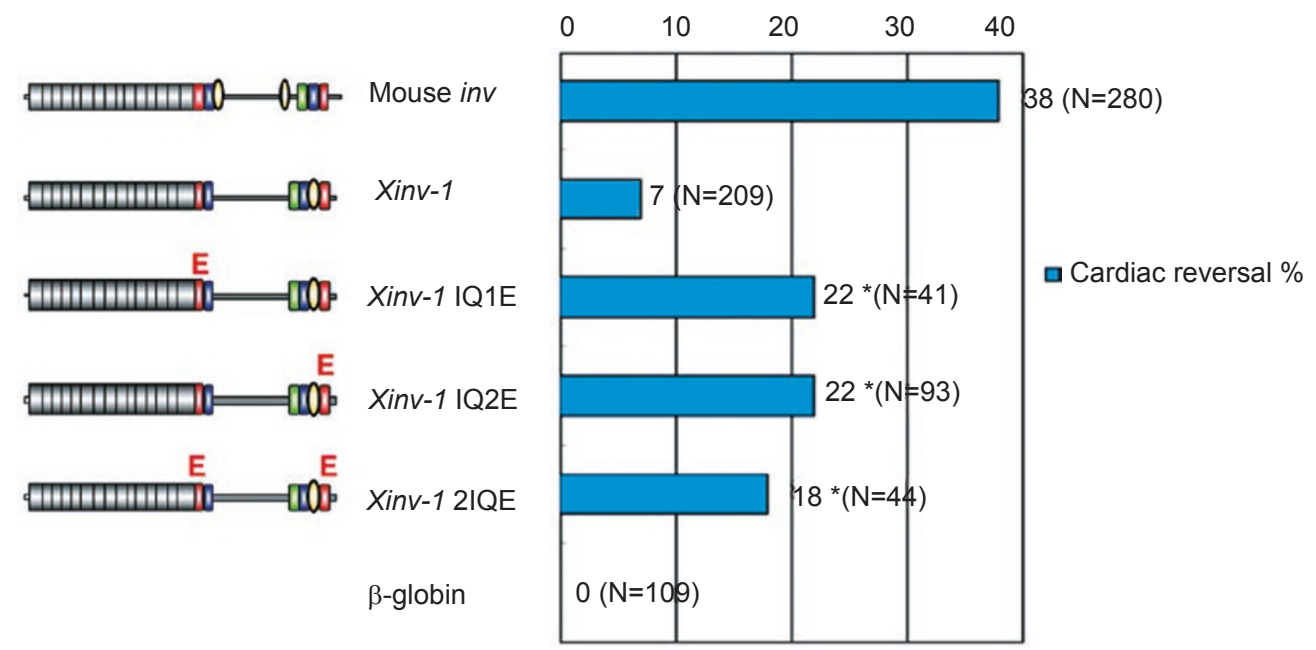

Figure 6 IQ-mutated but not WT Xinv mRNA injection reverses cardiac orientation. Cardiac reversal in Xenopus embryos injected with the mRNAs coding WT ORF or mutated construct of Xinv-1. Schematic description of the constructs indicated in the left lane. The red letters 'E' over the IQ motifs (depicted as red blocks) represent the amino-acid substitution. In the right graph, the percentages of embryos with cardiac reversal are shown. All three mutant Xinv mRNAs affected L-R asymmetry through the right-sided microinjection of mRNA, while the wild-type Xinv mRNAs did not. *Statistically different from that of the group injected with the Xinv-1WT RNA into a right blastomere.

present study identified two Xenopus inv genes (Xinv-1 and -2), Xinv-2 mRNA was not detected by RT-PCR, suggesting that Xinv-1 is transcribed mainly during Xenopus development, and that Xinv expression patterns shown by whole-mount in situ hybridization (Figure 3) are likely to reflect the distribution of Xinv-1 mRNA.

A previous study showed that the injection of mouse inv mRNA into Xenopus two-cell-stage embryos reverses cardiac orientation [14], and suggested that calmodulin signalling was involved in the inv pathway for the establishment of L-R asymmetry. Unexpectedly, mRNAs encoding wild-type Xenopus inv proteins did not affect cardiac orientation when injected into Xenopus two-cell stage embryos. As the N-terminal ankyrin repeats are highly homologous, the difference between mouse and Xenopus invs on cardiac inversion is likely to lie in the C-terminal half of inv proteins, probably by the interaction between calmodulin and IQ motifs. IQ-mutated Xinv-1 mRNA randomized cardiac orientation and lost interaction with calmodulin (Figure 6), supporting this idea. The results suggest that the interaction with calmodulin also regulates the Xenopus inv function. The mutation in only one of the two IQ motifs eliminated the overall CaM binding activity of Xinv protein (Figure 6), suggesting an intramolecular mechanism controlling the interaction between calmodulin and inv protein.

Randomization of the L-R asymmetry was induced only when these inv mRNAs were injected into the right blastomere, whereas injection into the left blastomere was ineffective. Our results suggest a factor(s) that activates inv protein differently between two blastomeres. The present study suggests that calmodulin is an important factor in the control of inv function. Calmodulin is a conserved $\mathrm{Ca}^{2+}$-binding protein involved in a variety of cellular calcium-dependent signalling pathways [23-25]. $\mathrm{Ca}^{2+}$ regulates the interaction between calmodulin and inv protein [14]. As recent studies suggest that there is a difference in $\mathrm{Ca}^{2+}$ concentration between the right and left sides in mice [26] and chickens [27], $\mathrm{Ca}^{2+}$ might be a candidate as a factor in making the difference in cardiac reversal induced by inv mRNA injection between right and left Xenopus blastomeres.

\section{Acknowledgments}

We thank Dr A Takao for his continuous support and encouragement of this work. We thank Dr H Takeda for his support and encouragement for this work. We thank Dr JB Gurdon for providing pGFP/RN3P. We thank Dr H Saiga, and Dr S Kuraku for helpful discussions. This work was supported by an Open Research Grant from The Japan Research Promotion Society for Cardiovascular Diseases, Grant-in Aid for Scientific Research (C) and Scientific Research on Priority Area (A) to T.Y.

\section{References}

1 Kosaki K, Casey B. Genetics of human left-right axis malformations. Semin Cell Dev Biol 1998; 9:89-99. 
2 Supp DM, Brueckner M, Potter SS. Handed asymmetry in the mouse: understanding how things go right (or left) by studying how they go wrong. Semin Cell Dev Biol 1998; 9:77-87.

3 Nonaka S, Tanaka Y, Okada Y, et al. Randomization of left-right asymmetry due to loss of nodal cilia generating leftward flow of extraembryonic fluid in mice lacking KIF3B motor protein. Cell 1998; 95:829-837.

4 Okada Y, Nonaka S, Tanaka Y, et al. Abnormal nodal flow precedes situs inversus in iv and inv mice. Mol Cell 1999; 4:459468.

5 Nonaka S, Shiratori H, Saijoh Y, Hamada H. Determination of left-right patterning of the mouse embryo by artificial nodal flow. Nature 2002; 418:96-99.

6 Yokoyama T, Copeland NG, Jenkins NA, et al. Reversal of left-right asymmetry: a situs inversus mutation. Science 1993; 260:679-682.

7 Lowe LA, Supp DM, Sampath K, et al. Conserved left-right asymmetry of nodal expression and alterations in murine situs inversus. Nature 1996; 381:158-161.

8 Meno C, Saijoh Y, Fujii H, et al. Left-right asymmetric expression of the TGF beta-family member lefty in mouse embryos. Nature 1996; 381:151-155.

9 Okada Y, Takeda S, Tanaka Y, et al. Mechanism of nodal flow: a conserved symmetry breaking event in left-right axis determination. Cell 2005; 121:633-644.

10 Mochizuki T, Saijoh Y, Tsuchiya K, et al. Cloning of inv, a gene that controls left/right asymmetry and kidney development. Nature 1998; 395:177-181.

11 Morgan D, Turnpenny L, Goodship J, et al. Inversin, a novel gene in the vertebrate left-right axis pathway, is partially deleted in the inv mouse. Nat Genet 1998; 20:149-156.

12 Schön P, Tsuchiya K, Lenoir D, et al. Identification, genomic organization, chromosomal mapping and mutation analysis of the human INV gene, the ortholog of a murine gene implicated in left-right axis development and biliary atresia. Hum Genet 2002; 110:157-165.

13 Otto EA, Schermer B, Obara T, et al. Mutations in INVS encoding inversin cause nephronophthisis type 2, linking renal cystic disease to the function of primary cilia and left-right axis determination. Nat Genet 2003; 34:413-420.

14 Yasuhiko Y, Imai F, Ookubo K, et al. Calmodulin binds to inv protein: implication for the regulation of inv function. Dev Growth Differ 2001; 43:671-681.
15 Harland RM. In situ hybridization: an improved whole-mount method for Xenopus embryos. Methods Cell Biol 1991; 36:685695.

16 Rupp RA, Snider L, Weintraub H. Xenopus embryos regulate the nuclear localization of XMyoD. Genes Dev 1994; 8:13111323.

17 Zernicka-Goetz M, Pines J, Ryan K, et al. An indelible lineage marker for Xenopus using a mutated green fluorescent protein. Development 1996; 122:3719-3724.

18 Shibata M, Shinga J, Yasuhiko Y, et al. Overexpression of $S$ adenosylmethionine decarboxylase (SAMDC) in early Xenopus embryos induces cell dissociation and inhibits transition from the blastula to gastrula stage. Int J Dev Biol 1998; 42:675-686.

19 Nieuwkoop PD, Faber J. Normal Table of Xenopus laevis (Daudin). New York \& London: Garland Publishing Inc 1994:252.

20 Kozak M. Point mutations define a sequence flanking the AUG initiator codon that modulates translation by eukaryotic ribosomes. Cell 1986; 44:283-292.

21 Saitou N, Nei M. The neighbor-joining method: a new method for reconstructing phylogenetic trees. Mol Biol Evol 1987; 4:406425.

22 Yokoyama T, Shirayoshi Y, Murakami T, Nakatsuji N. Expression of the inv gene during development and intracellular localization of the inv protein. In: Takao A, Eds. Etiology and Morphogenesis of Congenital heart Disease: Twenty Years of Progress in Genetics and Developmental Biology. Futura Publishing Company: NY, 2000:15-18.

23 Crivici A, Ikura M. Molecular and structural basis of target recognition by calmodulin. Annu Rev Biophys Biomol Struct 1995; 24:85-116.

24 Chin D, Means AR. Calmodulin: a prototypical calcium sensor. Trends Cell Biol 2000; 10:322-328.

25 Jurado LA, Chockalingam PS, Jarrett HW. Apocalmodulin. Physiol Rev 1999; 79:661-682.

26 McGrath J, Somlo S, Makova S, et al. Two populations of node monocilia initiate left-right asymmetry in the mouse. Cell 2003; 114:61-73.

27 Raya A, Kawakami Y, Rodriguez-Esteban C, et al. Notch activity acts as a sensor for extracellular calcium during vertebrate left-right determination. Nature 2004; 427:121-128.

Edited by Yun-Bo Shi 